\title{
The ubiquitin-like modifier FAT10 in cancer development
}

\author{
Annette Aichem ${ }^{\mathrm{a}}$, Marcus Groettrup ${ }^{\mathrm{a}, \mathrm{b}, *}$ \\ a Biotechnology Institute Thurgau at the University of Konstanz, CH-8280 Kreuzlingen, Switzerland \\ ${ }^{\mathrm{b}}$ Division of Immunology, Department of Biology, University of Konstanz, D-78457 Konstanz, Germany
}

\begin{abstract}
A B S T R A C T
During the last years it has emerged that the ubiquitin-like modifier FAT10 is directly involved in cancer development. FAT10 expression is highly up-regulated by pro-inflammatory cytokines IFN- $\gamma$ and TNF- $\alpha$ in all cell types and tissues and it was also found to be up-regulated in many cancer types such as glioma, colorectal, liver or gastric cancer. While pro-inflammatory cytokines within the tumor microenvironment probably contribute to FAT10 overexpression, an increasing body of evidence argues that pro-malignant capacities of FAT10 itself largely underlie its broad and intense overexpression in tumor tissues. FAT10 thereby regulates pathways involved in cancer development such as the NF- $\mathrm{KB}$ - or Wnt-signaling. Moreover, FAT10 directly interacts with and influences downstream targets such as MAD2, p53 or $\beta$-catenin, leading to enhanced survival, proliferation, invasion and metastasis formation of cancer cells but also of non-malignant cells. In this review we will provide an overview of the regulation of FAT10 expression as well as its function in carcinogenesis.
\end{abstract}

\section{Contents}

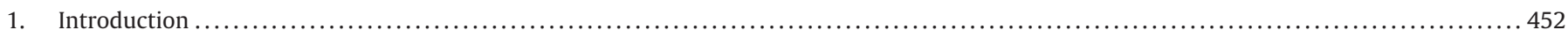

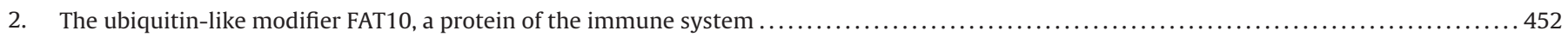

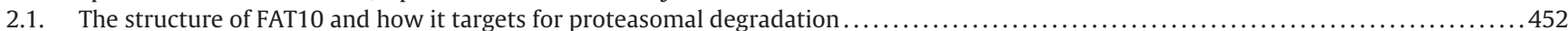

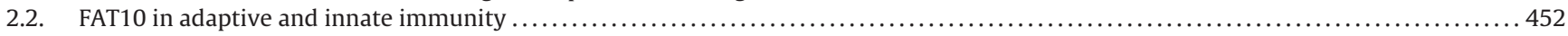

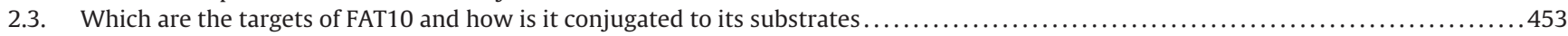

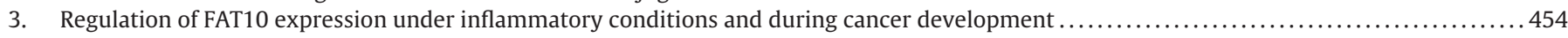

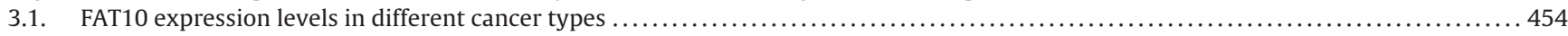

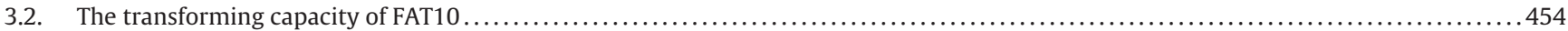

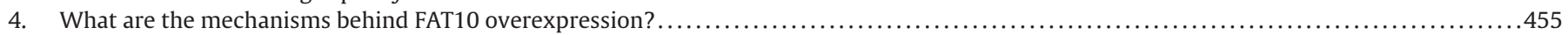

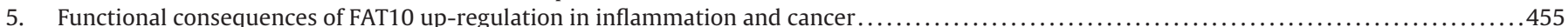

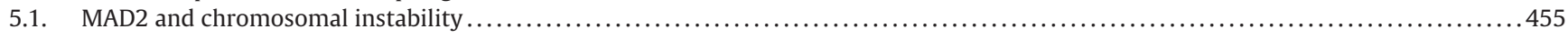

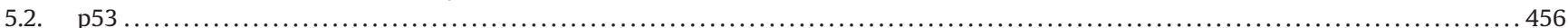

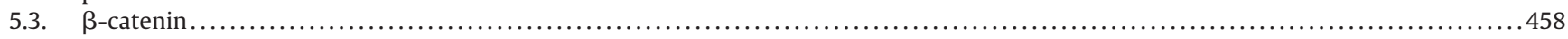

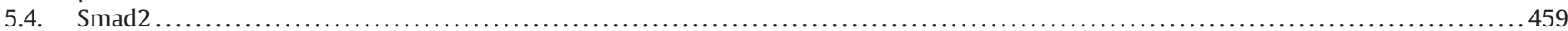

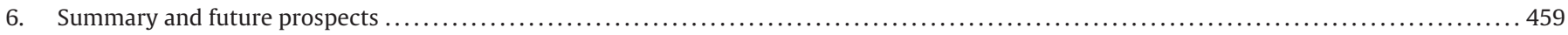

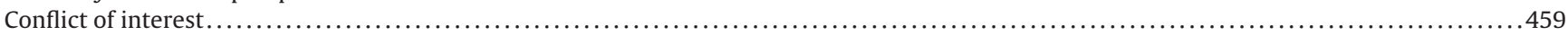

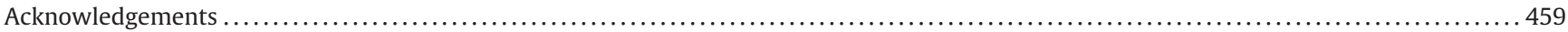

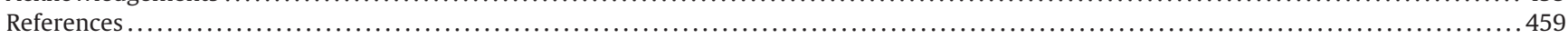

Abbreviations: AIPL1, aryl-hydrocarbon receptor like-1; APC/C, anaphase-promoting complex/cyclosome; DUB, deubiquitylating enzyme; EMT, epithelial-mesenchymal transition; FAT10, HLA-F adjacent transcript 10; GSK, glycogen synthase kinase; HCC, hepatocellular carcinoma; HLA, human leukocyte antigen; HOX, homeobox; IFN, interferon; IL, interleukin; MAD2, mitotic arrest deficient 2; mTEC, medullary thymic epithelial cells; NF-кB, nuclear factor kappa-B; NUB1L, NEDD8-ultimate buster 1 long; STAT, signal transducer and activator of transcription; SUMO, small-ubiquitin-like modifier; TGF, transforming growth factor; TNF, tumor necrosis factor; UBD, Ubiquitin D; ULM, ubiquitin-like modifier; UPS, ubiquitin proteasome system; USE1, UBA6-specific E2 enzyme 1.

* Corresponding author at: Division of Immunology, Department of Biology, University of Konstanz, Universitaetsstr. 10, D-78457 Konstanz, Germany.

E-mail address: marcus.groettrup@uni-konstanz.de (M. Groettrup). 


\section{Introduction}

A causal link between chronic inflammation or infection and tumorigenesis is nowadays a generally accepted principle leading to the development of various cancer types. The underlying mechanism is in part based on the upregulation and secretion of several proinflammatory cytokines such as tumor necrosis factor (TNF) $\alpha$, interferon (IFN) $\gamma$ or interleukin-6 (IL-6) by stromal cells as well as by infiltrating immune cells in the tumor microenvironment, or by cytokines, secreted by tumor cells themselves (Zamarron and Chen, 2011). Such inflammatory conditions are generated to promote healing and regeneration of the affected tissue. However, the persisting expression of these cytokines can also provide a niche for cancer development by promoting DNA damage and abnormal tissue healing. This is based on the fact that proinflammatory cytokines cause the activation of transcription factors such as nuclear factor kappa-B (NF- $\kappa \mathrm{B})$ and signal transducer and activator of transcription (STAT)3, which play a central role as activators of anti-apoptotic gene expression and cell proliferation. Furthermore, these cytokines promote optimal conditions for tumor cell proliferation, invasion and metastasis formation, apoptosis resistance as well as angiogenesis, i.e. processes which are all prerequisites for cancer development (Grivennikov et al., 2010; Karin, 2006).

The reversible post-translational modification of proteins with a single ubiquitin or with ubiquitin chains is an important mechanism to regulate the function, activity, cellular distribution but also the stability of proteins by mediating their proteolysis by the $26 \mathrm{~S}$ proteasome. The ubiquitin proteasome system (UPS) is not only an important regulator of protein degradation but also regulates cancer-relevant processes such as cell cycle progression, proliferation, DNA damage response, angiogenesis and apoptosis (Ciechanover, 1998; Haglund and Dikic, 2005). Moreover, inhibitors of the proteasome are already in use or under further investigation for the development of cancer therapies (Chen and Dou, 2010; Tu et al., 2012). Ubiquitylation as well as poly-ubiquitylation is achieved by an enzymatic cascade where first ubiquitin is adenylated at its C-terminal diglycine residue by one of its two ubiquitin E1 activating enzymes UBE1 (Ciechanover et al., 1981) or UBA6 (Jin et al., 2007; Pelzer et al., 2007) and then transferred onto the active site-cysteine of the respective E1 enzyme to form a thioester bond. In a second step, ubiquitin is transferred to the active site cysteine of a cognate ubiquitin conjugating enzyme (E2) by a transthiolation reaction. Finally, different classes of ubiquitin ligases (E3s) catalyse the isopeptide linkage of ubiquitin to the $\varepsilon$-amino-group of an internal lysine residue of a substrate protein (Finley, 2009; Kerscher et al., 2006). Ubiquitin itself contains 7 lysine residues (at positions $6,11,27,29,33,48$ and 63 ) which are used to build up chains of different linkage types conveying different outcomes for such modified proteins. Modification of a protein with a single ubiquitin is not enough to guide proteins to proteasomal degradation but requires the attachment of a ubiquitin chain of at least four ubiquitin molecules, linked through one of the above mentioned internal lysine residues. All different linkage types, with the exception of $\mathrm{K} 63$, lead to the degradation of the modified protein by the 26S proteasome (Xu et al., 2009), a barrel shaped multi-enzyme complex, consisting of a 20S core particle and one or two 19S regulatory particles (Finley, 2009). Before degradation, ubiquitin is removed from the proteins by so called deubiquitylating enzymes (DUBs) and recycled (Reyes-Turcu et al., 2009; Wolberger, 2014).

Besides ubiquitin, several ubiquitin-like modifiers (ULMs) have been identified. The ULMs family includes Human Leukocyte Antigen (HLA)-F adjacent transcript 10 (FAT10), small-ubiquitin-like modifier (SUMO)-1/2/3, ISG15, NEDD8, ATG8, ATG12, UFM-1, and URM-1. The ULMs share a similar three-dimensional structure, the ubiquitin or $\beta$-grasp fold, as well as the $\mathrm{C}$-terminal diglycine motif for activation and covalent attachment to substrate proteins by an E1-E3 enzymatic cascade (Hochstrasser, 2009). Of all these ULMs, FAT10 is the only modifier which acts as an autonomous transferable signal for degradation by the $26 \mathrm{~S}$ proteasome and it was shown that this process can occur independently of ubiquitin (Hipp et al., 2005; Schmidtke et al., 2009). FAT10 is a protein of the immune system and it is strongly up-regulated by pro-inflammatory cytokines (Fan et al., 1996; Raasi et al., 1999) and during the maturation of antigen presenting cells (Buerger et al., 2015; Ebstein et al., 2009; Lukasiak et al., 2008). Moreover, numerous recent publications point to a direct involvement of FAT10 in cancer development. These recent insights will be presented in this review along with the current state of knowledge on the regulation and function of FAT10 expression in inflammation and tumorigenesis (Fig. 1).

\section{The ubiquitin-like modifier FAT10, a protein of the immune system}

\subsection{The structure of FAT10 and how it targets for proteasomal degradation}

The Fat10 gene (originally designated Ubiquitin D (Ubd)) was identified by genomic sequencing of the human major histocompatibility complex (MHC) by Sherman Weissman and colleagues in 1996 (Fan et al., 1996). The structural model of the 165 amino acid long, $18 \mathrm{kDa}$ protein FAT10 as well as a previously published NMR structure of the N-terminal ubiquitin-like domain of FAT10 predicts two ubiquitin-like domains, both with a typical $\beta$-grasp fold. The Nand C-terminal ubiquitin-like domains are $29 \%$ and $36 \%$ identical to ubiquitin, respectively, arranged in a tandem array and separated by a short linker (Fan et al., 1996; Groettrup et al., 2008; Theng et al., 2014). In contrast to ubiquitin and other ubiquitin-like modifiers which are expressed as inactive precursors and which need to be activated by proteolytic processing at their C-terminus by specific proteases (Kerscher et al., 2006), FAT10 is already synthesized as a mature protein with a free diglycine motif at its $\mathrm{C}$-terminus and can directly be activated and conjugated to substrate proteins (Raasi et al., 2001). A central function of FAT10 modification is to target proteins for degradation by the proteasome (Hipp et al., 2005; Raasi et al., 2001; Schmidtke et al., 2014). This process was shown to be independent of ubiquitylation but to be dependent on a noncovalent interaction partner of FAT10, namely NEDD8-ultimate buster 1 long (NUB1L) (Hipp et al., 2005; Schmidtke et al., 2009, 2006). NUB1L, a likewise interferon-inducible protein (Kito et al., 2001), acts as a soluble receptor and brings FAT10 and FAT10ylated proteins to the proteasome where it binds to the 19S regulatory subunits RPN10 (S5a) and RPN1 (S2). It was shown that FAT10 itself could also directly bind to the VWA domain of RPN10 in the absence of NUB1L but that the docking of NUB1L to the proteasome was necessary for the accelerated degradation of FAT10 (Rani et al., 2012; Schmidtke et al., 2009, 2006). In contrast to ubiquitin, FAT10 has a very short half life of about $1 \mathrm{~h}$ and seems to be degraded by the proteasome along with its substrates (Aichem et al., 2014; Hipp et al., 2005). Since NUB1L negatively influences the stability of FAT10, the FAT10 degradation rate might be different in different cell lines or tissues expressing different amounts of NUB1L, as e.g. in HEK293 cells, which express only low amounts of NUB1L and where FAT10 is more stable (Aichem et al., 2014). In line with this, no FAT10-deconjugating enzymes, which might contribute to FAT10 recycling, have been identified to date.

\subsection{FAT10 in adaptive and innate immunity}

The FAT10 gene is encoded in the MHC class I locus and the highest FAT10 mRNA expression is measured in organs of the immune 


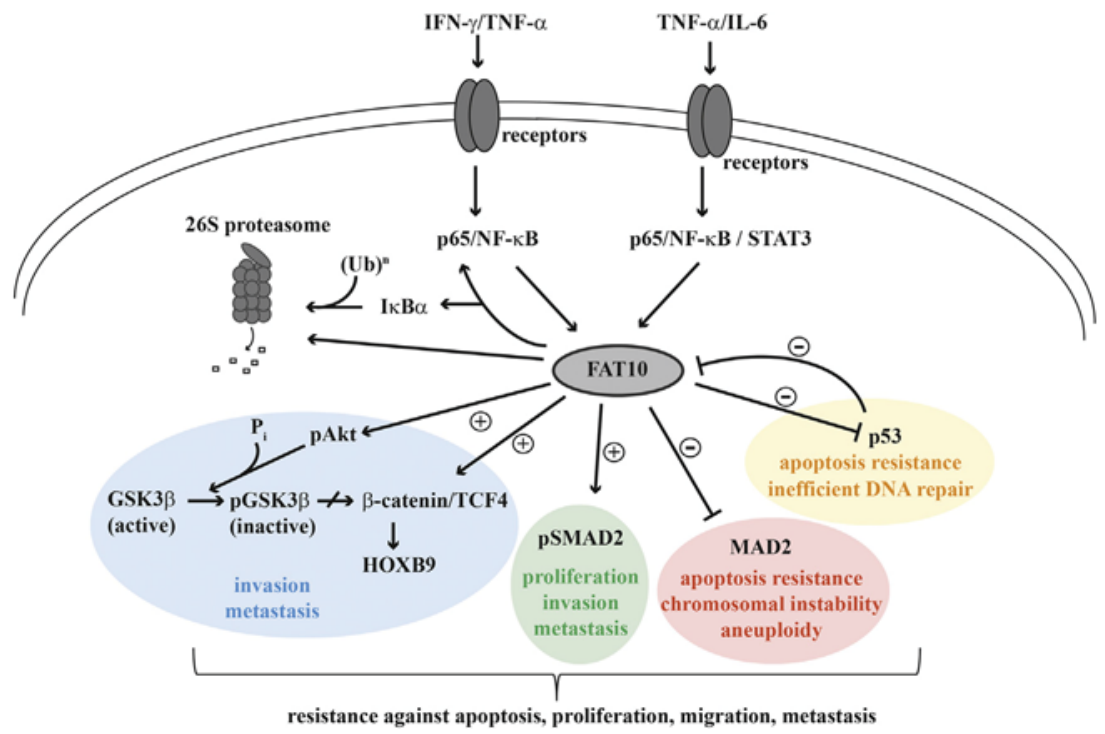

Fig. 1. Scheme showing the regulation of FAT10 expression as well as downstream targets which are influenced by FAT10 overexpression in tumor tissues.

The broad and intense overexpression of FAT10 in tumor tissues, induced by pro-inflammatory cytokines such as IFN- $\gamma$, TNF- $\alpha$ and IL- 6 within the tumor microenvironment, contributes to the malignant capacities of FAT10 itself. FAT10 regulates pathways involved in cancer development such as NF- $\kappa$ B-, Akt-, or Wnt-signaling and also directly interacts with and influences downstream targets such as p53, $\beta$-catenin, SMAD2, and MAD2, leading to enhanced survival, proliferation, invasion and metastasis formation of cancer cells but also of non-malignant cells. Arrows marked with a "+" illustrate an activating pathway, arrows marked with a "-" illustrate negatively regulated pathways.

system such as thymus, lymph nodes, fetal liver and spleen (Canaan et al., 2006; Lee et al., 2003; Liu et al., 1999; Lukasiak et al., 2008). The high expression of FAT10 in the thymus could be allocated to medullary thymic epithelial cells (mTECs) and it was shown that FAT10 modifies thymic T-cell selection, probably due to an altered peptide presentation on MHC class I molecules (Buerger et al., 2015). Additional data point to a role of FAT10 in MHC class I antigen presentation. Ebstein and colleagues (Ebstein et al., 2012) showed that FAT10 could enhance the MHC class I presentation of the human cytomegalovirus (HCMV)-derived antigen pp65 in HeLa cells by about two-fold as compared to untagged pp65 and Schliehe and colleagues (Schliehe et al., 2012) showed that the degradation rate as well as the presentation of specific lymphocyte choriomeningitis virus (LCMV) epitopes on MHC class I molecules was enhanced when FAT10 was fused to the N-terminus of the LCMV nucleoprotein. Apart from its expression in mTECs FAT10 is also constitutively expressed in mature B cells and dendritic cells (Bates et al., 1997) and its expression is highly and synergistically inducible on mRNA and protein level in numerous tissues and cell types by the pro-inflammatory cytokines IFN $\gamma$ and TNF $\alpha$ (Aichem et al., 2010; Raasi et al., 1999). A recent publication showed, that a high induction of FAT10 expression is also achieved by a synergistic treatment of HepG2 cells with TNF- $\alpha$ in combination with IL-6 (Choi et al., 2014). Upregulation of FAT10 expression induced caspase-dependent apoptosis in HeLa cells, renal tubular epithelial cells as well as mouse fibroblasts (Liu et al., 1999; Raasi et al., 2001; Ross et al., 2006). However, the role of FAT10 in apoptosis is contradictory because other publications showed a pro-survival role of FAT10 (Canaan et al., 2006). Lymphocytes of FAT10 knock out mice were shown to be more susceptible to spontaneous cell death than lymphocytes from wild type mice and human and mouse cardiac myocytes were protected from apoptosis induced by myocardial infarction associated hypoxia/reoxygenation stress through up-regulation of FAT10 (Peng et al., 2013).

Next to the role of FAT10 in adaptive immune responses there are recent publications which assign a function in innate immunity to FAT10. FAT10 was shown to play a role in the intracellular defense against bacteria (Spinnenhirn et al., 2014). Intracellular Salmonella Typhimurium which were targeted for xenophagy by being decorated with ubiquitin, the autophagy receptors p62 and NDP52 as well as with LC3 were simultaneously decorated with FAT10. Spinnenhirn and colleagues (Spinnenhirn et al., 2014) showed that the presence of FAT10 resulted in a prolonged life span of S. Typhimurium-infected mice, pointing to a protective role of FAT10. It is assumed that FAT10 plays additional roles in innate immunity since an up-regulation of FAT10 expression was described in HIV-infected renal tubular epithelial cells (RTECs) (Ross et al., 2006; Snyder et al., 2009) and during Kaposi sarcomaassociated herpesvirus infection (Hong et al., 2004). Moreover, the group of J.-Y. Yoo showed that FAT10 interfered with RIG-I (retinoic acid inducible gene 1) mediated antiviral signaling necessary for the production of type I interferons. By non-covalently interacting with RIG-I, FAT10 sequestered RIG-I into insoluble aggregates and this resulted in a diminished anti-viral immune response (Nguyen et al., 2016).

\subsection{Which are the targets of FAT10 and how is it conjugated to its substrates}

Upon IFN- $\gamma /$ TNF- $\alpha$ mediated induction of FAT10 in the human embryonic kidney cell line HEK293, the FAT10 mRNA was detectable already after $4-5 \mathrm{~h}$, however, FAT10 protein was prominently detected in western blots only $24 \mathrm{~h}$ after cytokine stimulation with the highly sensitive monoclonal antibody $4 \mathrm{~F} 1$ (Aichem et al., 2012, 2010). Nevertheless, upon immunoprecipitation of FAT10 and its modified substrates with mAb 4F1, combined with a subsequent western blot analysis with a polyclonal FAT10 specific antibody, bulk FAT10 conjugates of a large range of sizes are detectable, similar to ubiquitin, ISG15 or SUMO conjugates. A mass spectrometry analysis identified 571 putative FAT10 interaction partners, of which 176 proteins were classified as putative conjugation substrates and the remaining 395 proteins as non-covalent interaction partners. Interestingly, these proteins did not belong to distinct cellular pathways but covered a lot of different cellular functions, showing that FAT10 might play a role in numerous cell biological processes (Aichem et al., 2012; Leng et al., 2014). Of importance is that FAT10 apparently is not only functional when covalently conjugated to its substrate proteins, such as the ubiqui- 
tin activating enzyme UBE1 (Bialas et al., 2015; Rani et al., 2012), but also when interacting non-covalently with interaction partners such as HDAC6 (Kalveram et al., 2008), aryl-hydrocarbon receptor like-1 (AIPL1), an important protein within the retina (Bett et al., 2012), or the autophagosomal receptor p62/SQSTM1 which interacts both, covalently and non-covalently with FAT10 (Aichem et al., 2012). Activation and conjugation of FAT10 to its substrate proteins is performed most probably by a three step enzymatic cascade, as described for ubiquitin. The FAT10 E1 activating enzyme UBA6 acts as a bi-specific E1 enzyme, because it is able to activate both, FAT10 and ubiquitin (Chiu et al., 2007; Pelzer et al., 2007). In doing so, UBA6 is able to distinguish between the two modifiers and binds FAT10 with a higher affinity than ubiquitin, although the adenylation and transthiolation reaction is slower for FAT10 than for ubiquitin (Gavin et al., 2012). The activated FAT10 is then transferred onto the active site cysteine of the likewise bi-specific E2 conjugating enzyme UBA6-specific E2 enzyme 1 (USE1, also called UBE2Z) which directly undergoes auto-FAT10ylation in cis, mainly on Lys323 (Aichem et al., 2014, 2012; Gu et al., 2007; Jin et al., 2007). A portion of the auto-FAT10ylated USE1 is subsequently degraded by the proteasome, albeit the remaining USE1-FAT10 conjugate remains active with respect to FAT10 and ubiquitin transfer. Therefore, this mechanism might represent a negative feedback loop to limit the FAT10 conjugation pathway (Aichem et al., 2014). So far no FAT10-specific E3 ligases have been identified, but since all ULMs which get covalently conjugated to substrates need such ligases to be finally transferred onto their substrates, it is assumed that such E3 ligases must also exist for FAT10.

\section{Regulation of FAT10 expression under inflammatory conditions and during cancer development}

\subsection{FAT10 expression levels in different cancer types}

FAT10 expression is highly up-regulated in many different cancer types such as colon cancer, hepatocellular carcinoma (HCC), gastrointestinal and pancreatic carcinomas, gynecological carcinomas, and glioma (Lee et al., 2003; Lukasiak et al., 2008; Qing et al., 2011; Sun et al., 2014; Yuan et al., 2012). In liver neoplasia FAT10 is even described as an epigenetic marker (French et al., 2010a,b; Oliva et al., 2008). Moreover, in several cancer types a statistically significant association between a high FAT10 expression level and the progression and severity of the disease including a higher propensity for metastasis formation and poor prognosis, has been reported. These include triple-negative breast cancer (Han et al., 2015), glioma (Dai et al., 2016; Yuan et al., 2012), pancreatic ductal adenocarcinoma (Sun et al., 2014), HCC (Liu et al., 2014), or gastric cancer (Ji et al., 2009). Furthermore, colon cancer patients treated with a postoperative chemotherapy and bearing tumors with high FAT10 expression displayed a higher recurrence rate than those with FAT10-negative tumors and FAT10 expression was associated with a significantly shorter survival time (Zhao et al., 2015).

\subsection{The transforming capacity of FAT10}

Lukasiak et al. (2008) showed that the high upregulation of FAT10 expression in cancers of liver and colon strongly correlated with the expression of the proteasome subunit LMP2 which is induced in these organs by pro-inflammatory cytokines IFN- $\gamma$ and TNF- $\alpha$ in the tumor microenvironment. By performing a colony formation assay with FAT10 transfected NIH3T3 cells, no evidence for colony formation and transformation capacity of FAT10 was observed, arguing against a function of FAT10 as a proto-oncogene in this experimental model. Therefore it was questioned whether FAT10 has transforming capacity and whether such a property could explain its overexpression in these cancers. However, FAT10 overexpression in the immortalized liver cell line NeHepLxHT could confer malignant properties to these otherwise non-tumorigenic cells. NeHepLxHT cells, overexpressing clinically relevant amounts of FAT10, showed a dose-dependent effect of FAT10 on proliferation, anchorage independent growth - a hallmark of transformed malignant cells - and resistance to apoptosis (Gao et al., 2014). In addition, as compared to control cells, FAT10 expressing NeHepLxHT cells significantly increased their invasive capacity across matrigel as well as their migratory capacity in a scratch-woundhealing assay, again in a FAT10 dose-dependent manner. The same results were obtained when using a second cell line stably transfected with a FAT10 expression plasmid, namely the tumorigenic colorectal cancer cell line HCT116. FAT10 expressing NeHepLxHT cells induced tumor formation, again in a dose-dependent manner, when inoculated into nude mice as compared to mice, which had been treated with empty vector-transfected, FAT10-negative control cells. Furthermore, FAT10 overexpression in HCT116 cells even greatly enhanced tumor growth as compared to FAT10-negative HCT116 cells, strongly arguing for a direct involvement of FAT10 in tumor formation (Gao et al., 2014). Therefore it seems that FAT10 cannot only confer malignant properties to non-tumorigenic cells but that it can even augment the malignancy associated traits of a cancer cell line.

Additional data also suggested FAT10 to be a proto-oncogene: knockdown of FAT10 in the FAT10-positive hepatocellular carcinoma cell line Hep3B specifically inhibited cell proliferation and colony formation under in vitro conditions, most probably due to a blockage of the entry into the S-phase of the cell cycle and by inducing apoptosis, as measured by AnnexinV staining. Under in vivo conditions using nude mice which had received a subcutaneous injection of Hep3B cells it was further shown that the Hep3B xenograft growth was much reduced when the mice were treated with an intratumoral injection of Ad-siRNA/FAT10 two weeks after injection of the Hep3B cells. The knockdown of FAT10 even prolonged the lifespan of these mice as compared to mice, which had been treated with a control virus or PBS (Chen et al., 2014).

The notion that FAT10 is directly involved in the development of tumors is at least consistent with recent investigations of FAT10 gene knock out mice. These mice are viable and fertile and exhibit only minimal phenotypic differences to their wild type counterparts such as e.g a higher sensitivity towards endotoxin challenge. In addition, as already mentioned, lymphocytes from these mice are more prone to spontaneous apoptotic cell death (Canaan et al., 2006). Noteworthy, when examining older FAT10-deficient mice, it could be observed that these mice showed delayed aging and an appox. 20\% increase in life span. This phenotype was accompanied with a significant reduction in adipose tissue and an increased metabolism in muscle tissue. In muscles of FAT10 deficient mice, decreased levels of pro-inflammatory cytokines and enhanced levels of anti-inflammatory cytokines such as IL-10 were measured, thereby creating an inflammation-suppressive milieu in skeletal muscle and blood which may account for the absence of tumors in these mice (Canaan et al., 2014).

Taken all these findings together, several publications support the notion that FAT10 itself is a proto-oncogene and that its upregulation during inflammatory processes is involved in the development of different cancer types. This conception poses further questions: (1) How is the expression of FAT10 regulated during inflammation and cancer? (2) Which are the downstream targets of FAT10? and (3) How does FAT10 enhance the malignant properties of these proteins. 


\section{What are the mechanisms behind FAT10 overexpression?}

What are the molecular mechanisms how FAT10 exerts its malignant effects? One potential mechanism could be based on a recently described involvement of FAT10 in NF-кB signaling (Fig. 1). NF- $\kappa \mathrm{B}$ is an important transcription factor for the regulation of genes involved in inflammation, proliferation, cell-cycle control, apoptosis, and the immune response and plays therefore a critical role in carcinogenesis (Hoesel and Schmid, 2013). On the one hand FAT10 may act as a regulator of NF- $\kappa$ B signaling and on the other hand FAT10 expression itself is regulated by NF- $\kappa$ B. As already pointed out, FAT10 mRNA and protein expression is highly and synergistically inducible in all cell types by the pro-inflammatory cytokines IFN- $\gamma$ and TNF- $\alpha$ (Aichem et al., 2010; Raasi et al., 1999; Ren et al., 2011) or by a combination of TNF- $\alpha$ and IL- 6 as shown in HepG2 cells (Choi et al., 2014). In HCT116 cells it was shown, that TNF- $\alpha$ signaling via the TNF- $\alpha$ receptor 1 (TNFR1) induced FAT10 expression, a receptor mainly found on tumor and stromal cells where it mediates the activation of NF- $\mathrm{BB}$ (Balkwill, 2009; Ren et al., 2011). The analysis of the FAT10 promoter sequence revealed seven potential p65/NF- $\mathrm{BB}$ binding sites as well as three binding sites for transcription factors of the family of signal transducer and activator of transcription (STAT)3 (Canaan et al., 2006; Choi et al., 2014; Gao et al., 2015). NF-кB and STAT3 cooperatively regulate a number of target genes and it was shown that binding of one of these transcription factors to the respective promoter sequence facilitates recruitment of the other, and vice versa. STAT3 activation is mediated mainly by IL-6 and this leads to the expression of genes involved in cell proliferation, angiogenesis and survival. STAT3 is constitutively active in many cancer types such as breast and colon cancer and its expression level positively correlates with the aggressiveness of the tumor (Bosch-Barrera and Menendez, 2015; Hoesel and Schmid, 2013; Wake and Watson, 2015). Choi et al. (2014) have shown that STAT3 and p65/NF-кB act synergistically to activate FAT10 expression upon IL- 6 and TNF- $\alpha$ induction. Further support for the regulation of FAT10 expression by NF- $\mathrm{B}$ signaling is derived from experiments with a substance called silibinin, a flavonoid isolated from seed extracts of the herb milk thistle (Silybum marianum). Silibinin exhibits several potent anti-cancer properties such as acting as an antioxidant, having immunomodulatory and anti-proliferative properties and it was shown to inhibit multiple cancer signaling pathways as for instance NF- $\kappa \mathrm{B}$ signaling (Bosch-Barrera and Menendez, 2015; Singh et al., 2004; Tyagi et al., 2012). Caroline Lee and colleagues found that upon treatment of IFN- $\gamma /$ TNF- $\alpha$-stimulated HCT116 cells with increasing amounts of silibinin, the FAT10 mRNA expression was diminished in a dose-dependent manner and this was due to a silibinin-mediated prevention of the nuclear translocation of p65 most probably due to an inhibition of the degradation of the NF- $\kappa$ B inhibitor I $\kappa \mathrm{B} \alpha$ (Gao et al., 2015). Tumor formation by TNF- $\alpha$-induced and thus FAT10 expressing HCT116 cells in nude mice was diminished when the mice were treated at the same time with silibinin which correlated with reduced FAT10 expression in the tumors. The dependency of endogenous FAT10 expression on NF- $\mathrm{kB}$ signaling was further supported by data showing that the induction of FAT10 expression by TNF- $\alpha$ was greatly diminished when $\mathrm{p} 65$ was downregulated by specific siRNAs in HCT116 and HepG2 cells (Gao et al., 2015; Ren et al., 2011).

The interplay between FAT10 and NF- $\kappa$ B may not be a oneway street since in a previous study using renal tubular epithelial cells, FAT10 was reported to activate NF- $\kappa$ B signaling, pointing to a potential positive feedback loop. Consistently, the TNF$\alpha$-induced Iк $\mathrm{B} \alpha$ degradation, as well as the expression of the NF- $\mathrm{BB}$-dependent genes CXCL2 and MCP-1 was impaired in FAT10deficient cells (Gong et al., 2010). Arguing against a RTEC cell type-confined effect of FAT10 on NF- $\mathrm{B}$ signaling, a microarray based transcriptome analysis of FAT10 overexpressing NeHepLxHT and HCT116 cells revealed in both cell lines that the NF-кB transcriptional activity was significantly enhanced as compared to control cells, not expressing FAT10. This was accompanied by a higher nuclear localization of NF- $\kappa \mathrm{B} / \mathrm{p} 65$, while the total cellular amount of p65 remained stable. At the same time, the amount of the NF- $\kappa B$ inhibitor I $\mathrm{B} \alpha$ was diminished in FAT10 overexpressing NeHepLxHT cells. A similar increase in nuclear p65 was observed in HCT116 cells, overexpressing FAT10. The increase in nuclear p65 could be reversed by knocking down FAT10 with FAT10-specific siRNAs (Gao et al., 2014). Taken together, it was shown that the increased FAT10 expression indirectly augmented NF- $\kappa B$ activity by alleviating the inhibition of NF- $\kappa \mathrm{B}$. NF- $\kappa \mathrm{B}$ is a transcription factor with multiple target genes, so the question arises, which are the genes downstream of FAT10 and NF- $\kappa$ B? The microarray expression analysis of FAT10 overexpressing NeHepLxHT and HCT116 cells by Gao et al. (2014) revealed that the chemokine receptors CXCR4 and CXCR7 were highly up-regulated in FAT10 overexpressing NeHepLxHT cells, consistent with other data showing that NF-кB is a transcription factor for both genes (Katoh and Katoh, 2010; Tarnowski et al., 2010). CXCR4 and -7 are both described to promote invasion and tumor growth in several cancer types (Gao et al., 2010; Heinrich et al., 2012; Liang et al., 2005; Zheng et al., 2010). In accordance with these data, an siRNA-mediated knockdown of p65 or overexpression of IкB $\alpha$ in FAT10 overexpressing NeHepLxHT cells significantly attenuated cell invasion and migration in vitro, precisely as silencing of CXCR4 and CXCR7 did. Taken together, these data point towards a mechanism where up-regulation of FAT10 leads to activation of NF- $\kappa B$ signaling which in turn activates the expression of CXCR4 and -7, resulting in enhanced migration and invasion of FAT10 overexpressing cells (Gao et al., 2014).

In summary, it emerges that FAT10 expression upon TNF- $\alpha /$ IFN$\gamma$ or TNF- $\alpha /$ IL- 6 stimulation is mediated mainly by NF- $\kappa B$ and STAT3 signaling. Thereby, a FAT10-dependent positive feedback loop seems to exist which enhances NF- $\kappa B$ activation and which may contribute to the malignant properties of FAT10. This directly leads to the next question, namely which proteins get targeted by FAT10 and what are the consequences of their interaction with FAT10 with respect to cancer development.

\section{Functional consequences of FAT10 up-regulation in inflammation and cancer}

\subsection{MAD2 and chromosomal instability}

Upregulation of endogenous FAT10 expression by TNF- $\alpha$ as well as overexpression of FAT10 has directly been linked to chromosomal instability in several reports (Gao et al., 2015; Ren et al., 2006, 2011; Theng et al., 2014). It was shown that about 60\% of HCT116 and HepG2 cells which had been treated for about ten passages with IFN- $\gamma$ and TNF- $\alpha$ displayed abnormally high chromosome numbers in a karyotype analysis and inhibition of FAT10 expression with silibinin, a potent inhibitor of the NF- $\mathrm{kB}$ signaling pathway, was able to abolish this effect (Gao et al., 2015). FAT10 was shown to be cell-cycle regulated with a peak of expression in S-phase but low expression levels during G2/M phase (Lim et al., 2006; Liu et al., 2014) and a proteomics based study provided evidence for a role of FAT10 in mitotic regulation (Merbl et al., 2013). Already 17 years ago MAD2 (Mitotic arrest deficient 2), a mitotic spindle checkpoint protein, was identified as a non-covalent interaction partner of FAT10 in a yeast two hybrid screen using a human B cell cDNA library (Liu et al., 1999) and this interaction was shown to prevail during mitosis (Ren et al., 2006). MAD2 binds to the kinetochores of sister chromatids in the metaphase of the cell cycle. There it ensures that before the onset of anaphase all chromo- 
A

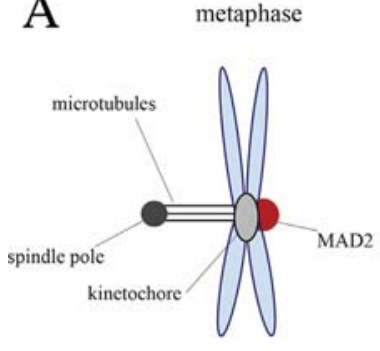

B

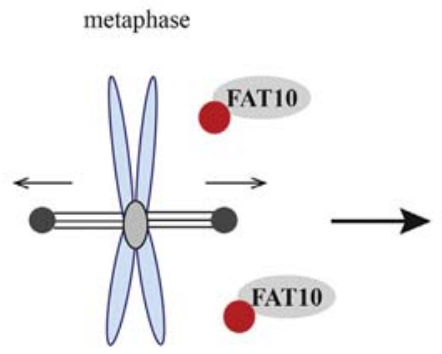

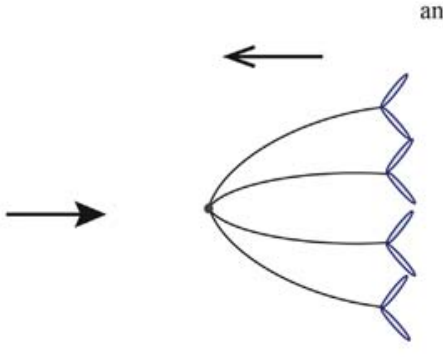

anaphase

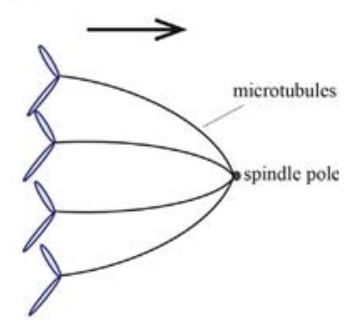

anaphase

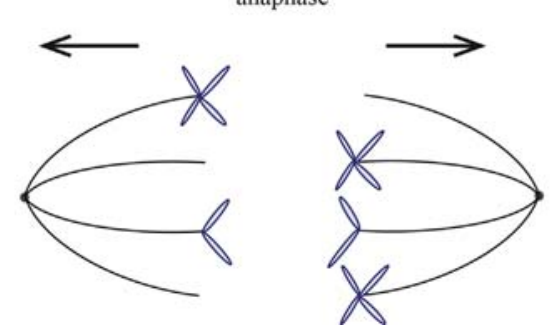

Fig. 2. Interaction of FAT10 with MAD2 induces a premature onset of anaphase, resulting in aneuploidy.

(A) Under normal conditions, the onset of anaphase and the separation of the sister chromatids is inhibited until all chromosomes are properly attached via their kinetochores to the mitotic spindle apparatus. This mechanism is surveilled by a protein complex containing MAD2. (B) Upregulation of FAT10 allows the non-covalent interaction of FAT10 with free MAD2 and in turn reduces the amount of MAD2 at the kinetochores. As a result, anaphase starts too early and results in inappropriate separation of sister chromatids and aneuploidy. A detailed description is given in the text.

somes become properly attached to the mitotic spindle along the metaphase plate (Fig. 2A). MAD2 achieves this by interacting with and inhibiting $\mathrm{Cdc} 20$, a co-activator and substrate recruitment factor of the anaphase-promoting complex/cyclosome (APC/C), which is a multi-subunit ubiquitin E3 ligase. Thereby the ubiquitylation of securin and cyclin is prevented. Securin binds and inhibits a protease called securase which cleaves cohesin, a protein that keeps the two sister chromatids together (Lara-Gonzalez et al., 2012; Musacchio and Salmon, 2007). In FAT10 overexpressing HCT116 cells as well as in TNF- $\alpha$-treated HCT116 cells, the localization of MAD2 at the kinetochores was much reduced in the prometaphase of the cell cycle, indicating that FAT10 interferes with the binding of MAD2 to kinetochores (Fig. 2B) (Ren et al., 2006, 2011). Moreover, after release from G1/S arrest, FAT10 overexpressing cells showed a delayed entry into mitosis. However, these cells also showed an unaltered reentry into G1 phase as compared to parental cells which points at an abbreviated mitotic phase or an abbreviated mitotic arrest upon spindle damage. When compared with control cells, a higher number of FAT10 overexpressing cells failed to arrest in mitosis upon treatment with nocadozole or even escaped the mitotic arrest. Concurrent with these findings, FAT10 overexpression induced the formation of multinucleated cells with abnormal nuclear morphology. A constitutive overexpression of FAT10 as well as constitutive treatment with TNF- $\alpha$ resulted in an increased chromosome number as compared to the parental HCT116 cell line (Ren et al., 2006, 2011). However, when the five putative interaction sites of MAD2 within the N-terminal ubiquitin-like domain of FAT10 were mutated (H11D, R13Q H75D, T77D and K79Q), binding of MAD2 to FAT10 was greatly diminished. As compared to cells overexpressing wildtype FAT10, the chromosome numbers of cells expressing the mutant FAT10 returned to normal as found in wild type HCT116 cells. This further supports the notion that FAT10 overexpression causes chromosomal instability by interacting with MAD2. Moreover, as compared to the parental cells, FAT10 overexpressing cells showed long, incompletely condensed chromosomes and this was also reverted back to normally condensed, short "ribbon-like" chromosomes in cells expressing the mutant
FAT10 deficient in MAD2 binding. In line with this observation, the inhibition of the FAT10 and MAD2 interaction also reverted the capability of FAT10 overexpressing cells to escape the nocadozoleinduced mitotic arrest back to levels, seen with wild type HCT116 cells (Theng et al., 2014). Taken together, these data corroborate the hypothesis that the non-covalent interaction of FAT10 and MAD2 is critical for the development of aneuploidy in cancer cells. It was shown that FAT10 interacts with free but not with spindle bound MAD2 as an immunoprecipitation against FAT10 led to a coimmunoprecipitation of MAD2 only, and not of additional proteins of the spindle complex such as MAD1 and Bub1 (Theng et al., 2014). The observed low level of endogenous FAT10 expression in G2/M phase (Lim et al., 2006) suggests that cells keep the FAT10 level low during the mitotic phase in order to prevent mitotic dysfunctions with the risk of subsequent cancer development. Consistently, HCT116 cells overexpressing the MAD2 interaction-deficient FAT10 mutant retained significantly lower proliferation rates and showed an increased susceptibility to apoptosis as compared to cells overexpressing wild type FAT10. Moreover, cells expressing the MAD2 binding-deficient FAT10 mutant failed to grow in soft agar and lost their capability to grow in an anchorage independent manner. More importantly, this finding could be substantiated in vivo by injecting HCT116 cells expressing FAT10 wildtype or the MAD2 binding-deficient FAT10 mutant into nude mice. Tumors grown from HCT116 cells expressing MAD2 binding-deficient FAT10 were significantly smaller than those expressing wild type FAT10 (Theng et al., 2014). In summary, all these data from well characterized cell line models of hepatocellular carcinoma make a convincing case, that the pro-malignant capacities of FAT10 rely extensively on its interaction with the spindle checkpoint protein MAD2, thereby preventing the proper function of MAD2 in mitotic checkpoint control (Fig. 2).

\section{2. $p 53$}

The transcription factor and tumor suppressor p53 plays a central role in the maintenance of genomic stability and as an inhibitor 


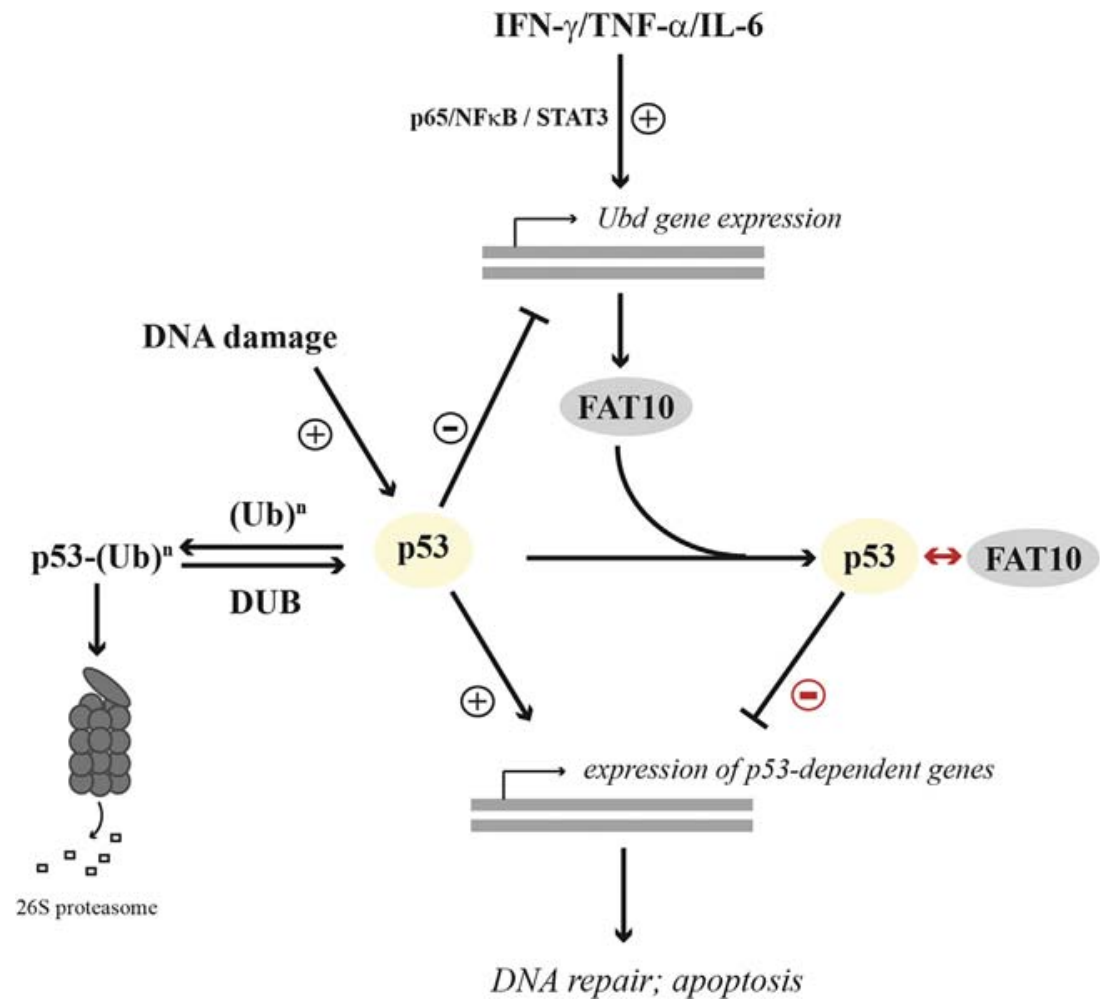

Fig. 3. FAT10 negatively regulates p53 transcriptional activity.

Under normal conditions, the concentration of p53 is kept at a low level and this is maintained by a tight regulation of p53 ubiquitylation and deubiquitylation by specific E3 ligases and deubiquitylation enzymes (DUBs). DNA damage leads to a rapid upregulation of p53, its activation by phosphorylation and its trans-localization into the nucleus where it acts either as an activator or as an inhibitor of transcription leading to DNA repair or induction of apoptosis. High FAT10 concentrations, mediated by pro-inflammatory cytokines lead to an inhibition of the p53 transcriptional activity and result in DNA damage and resistance to apoptosis. A detailed description is given in the text. Arrows marked with a "+" illustrate activating pathways, arrows marked with a "-" illustrate negatively regulated pathways. A red arrow with two arrowheads represents the interaction of $\mathrm{p} 53$ and FAT10.

of inflammation. More than $50 \%$ of all human cancers display deletions or mutations in the $p 53$ gene. Under normal conditions, the concentration of p53 is kept at a low level and this is maintained by a tight regulation of p53 ubiquitylation and deubiquitylation. Two main cellular events regulated by p53 are cell cycle arrest upon DNA damage and induction of apoptosis. DNA damage leads to a rapid up-regulation of $\mathrm{p} 53$, its activation by phosphorylation and its trans-localization into the nucleus where it acts either as an activator or as an inhibitor of transcription. Down-stream targets of p53 are genes involved in the regulation of DNA repair, apoptosis and cell cycle control. Upon up-regulation of p53, the cell cycle is arrested at the transition of G1/S until DNA repair is performed and the cell re-enters the cell cycle. However, if the DNA damage is too strong, apoptosis will be induced. Mutations in p53 which inhibit its activity as transcription factor are therefore linked to cancer development (Gudkov et al., 2011; Levine, 1997). p53 was recently identified as a FAT10 interacting protein in a mass spectrometry screen searching for FAT10 interaction partners (Aichem et al., 2012). Additional data showed that p53 gets covalently modified by overexpressed FAT10 in HEK293 cells, and that overexpression of wild type but not of mutant FAT10, in which the diglycine motif at the C-terminus was mutated, increased the transcriptional activity of a luciferase reporter construct, containing binding sides for p53 (Li et al., 2011). However, under inflammatory conditions the p53 transcriptional activity towards luciferase reporter constructs containing either an artificial p53-regulated promoter or a natural target promoter of p53 (p21 WAF1/CIP1) was decreased upon FAT10 induction by IL- 6 and/or TNF- $\alpha$. Consistently, overexpression of FAT10 in different cells lines also reduced the transcriptional activity of p53 without affecting the overall p53 protein amount. In support of this finding, silencing of FAT10 induced the opposite effect and led to an increase in the transcriptional activity of p53 and to an increase in apoptosis (Choi et al., 2014). The system seems to be tightly regulated by a negative feedback mechanism because it was shown, that p53 itself negatively regulates the expression of Fat10 mRNA by binding to an inhibitory site within the Fat10 promoter. It was shown that the transcriptional activity of $\beta$-galactosidase reporter constructs under the control of the Fat10 promoter was significantly higher in the p53 negative cell line Hep3B as compared to the p53 expressing cell lines HepG2 and KB3-1. Overexpression of p53 in Hep3B likewise decreased Fat 10 reporter activity as well as endogenous FAT10 expression. Conversely, when p53 was downregulated by specific siRNA in Hep3B cells overexpressing p53, Fat10 reporter activity increased. The same increase in endogenous FAT10 mRNA expression was obtained when endogenous p53 was downregulated by specific siRNA in HEK293 cells, clearly underlining that p53 negatively regulates the Fat 10 promoter. These data imply that mutant p53, as found in many tumors, would elevate FAT10 expression and along with it FAT10‘s pro-malignant capacities (Zhang et al., 2006). In support of this hypothesis, the mRNA and protein expression levels of FAT10 positively correlated with the mRNA and protein expression levels of mutant $\mathrm{p} 53$ in gastric cancer tissue and patients with high FAT10 expression in their tumors showed a tendency towards unfavorable prognosis regarding the overall survival rate (Ji et al., 2009).

Taken all these data together, one can construct a model of how the expression levels of p53 and FAT10 must be tightly regulated to prevent cells from becoming malignant (Fig. 3). In normal cells expressing functional p53, FAT10 expression is kept at a low 


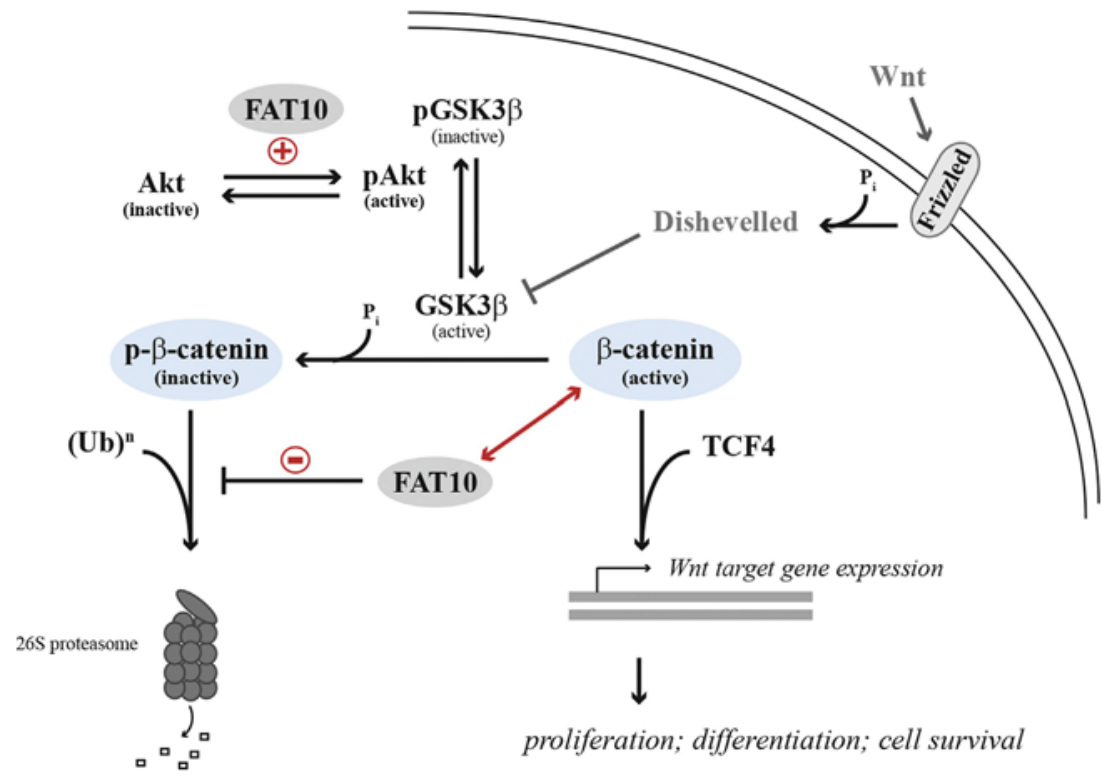

Fig. 4. FAT10 regulates $\beta$-catenin stability by influencing the Wnt and Akt signal transduction pathways.

The Wnt signaling pathway plays a critical role in promoting HCC carcinogenesis and metastasis. It leads to the activation of the transcription factor $\beta$-catenin by preventing its phosphorylation by GSK3 $\beta$ and thus its subsequent ubiquitylation and proteasomal degradation. FAT10 contributes to the malignant capacities of $\beta$-catenin by directly interacting with it and inhibiting its ubiquitin-dependent degradation by the proteasome. In a second line, upregulation of FAT10 leads to an increase of phosphorylated and thus activated Akt, which in turn phosphorylates and inactivates GSK3 $\beta$, which again results in stabilization of $\beta$-catenin. A detailed description is given in the text. An arrow marked with a "+" illustrates an activating pathway, an arrow marked with a "-" illustrates a negatively regulated pathway. Red arrow with two arrowheads represents the interaction of $\beta$-catenin and FAT10.

level due to the negative regulation of the FAT10 promoter by p53. In case of DNA damage, cell cycle arrest and DNA repair is performed to let the cell re-enter the cell cycle, or, if the DNA damage is too severe, apoptosis is induced. Chronic inflammatory conditions which induce high FAT10 expression lead to a transcriptional downregulation of $\mathrm{p} 53$-dependent gene expression and promote the interaction of FAT10 with MAD2. This leads to a too early onset of anaphase, chromosomal instability and protection from apoptosis. The presence of mutant, non-functional p53 might even enhance this effect and further support the transforming capacities of FAT10. The maintenance of the balance between FAT10 and p53 expression is therefore very important to protect cells from getting malignant.

\section{3. $\beta$-catenin}

The wnt signaling pathway leading to the activation of the transcription factor $\beta$-catenin plays a crucial role in the development of cancer. $\beta$-catenin, as long as it is not phosphorylated, can translocate into the nucleus where it forms heterodimers with other transcription factors such as T-cell factor and lymphoid enhancerbinding factor 1 , leading to the transcription of genes, involved in the regulation of proliferation, differentiation and cell survival (Fig. 4). Upon phosphorylation of $\beta$-catenin by the serine/threonine glycogen synthase kinase $3 \beta$ (GSK3 $\beta$ ) and by casein kinase I, $\beta$ catenin gets ubiquitylated by the E3 ligase $\beta$-TRCP and degraded by the proteasome (Amit et al., 2002; Polakis, 2000, 2012). Mutations in $\beta$-catenin which prevent its phosphorylation and subsequent ubiquitylation, and which result in a constitutive active transcription factor are found with significant frequency in HCC and it was shown that $\beta$-catenin plays a key role in the metastasis of human hepatocellular carcinoma (de La Coste et al., 1998; Lai et al., 2011). Recently, it was reported that the up-regulation of FAT10 expression promoted the invasion and metastasis of hepatocellular carcinoma cells by binding non-covalently to $\beta$-catenin and preventing its ubiquitylation and subsequent proteasomal degradation (Fig. 4). As a result, the expression of Homeobox B9 (HOXB9), a member of the class I homeobox (HOX) genes and a target of $\beta$-catenin/TCF4 (Hatzis et al., 2008), was found to be highly upregulated in HCC and the HOXB9 mRNA and protein expression levels correlated with the mRNA and protein expression levels of FAT10. HOX genes are normally involved in embryogenesis but a role in cancer development has been proposed (Abate-Shen, 2002). HOXВ9 regulates the expression of genes involved in the regulation of cell migration, invasion and metastasis formation. FAT10 and HOXB9 overexpression correlated with HCC tumor size, tumor microsatellite formation and vascular invasion, pointing to an involvement of both proteins in HCC aggressiveness. Taken together, these data show that FAT10 up-regulation stabilizes the $\beta$-catenin/TCF4 signaling pathway by preventing its ubiquitindependent proteasomal degradation, and this in turn induces an increase in HOXB9 expression, leading to HCC invasion and metastasis generation (Yuan et al., 2014).

Concurrent with these findings, FAT10 was found to influence the Akt/GSK3 $\beta$ signaling pathway (Fig. 4). The serine/threonine kinase Akt (or PKB) is a central node in cell signaling downstream of cytokines, growth factors and other stimuli. Akt signaling plays a role in regulating cell survival, differentiation, proliferation and angiogenesis, pointing to a role of the Akt signaling pathway in carcinogenesis (Manning and Cantley, 2007). One of the downstream targets of Akt is GSK3 $\beta$, which is inactivated when getting phosphorylated by Akt, and signal transduction via Akt/GSK3 $\beta$ was shown to promote TNF- $\alpha$-mediated epithelial-mesenchymal transition (EMT) (Wang et al., 2013). FAT10 overexpressing HCC cell lines HepG2 and SMMC-7721 were shown to undergo EMT because their morphology changed towards spindle-like fibroblastic cells, the expression of the epithelial marker E-cadherin was decreased, and the expression of the mesenchymal marker $\mathrm{N}$-cadherin and vimentin were increased. In addition, the amount of snail, an Ecadherin transcriptional repressor, was elevated in these cells. Further investigations showed that FAT10 mediated this effect via the Akt/GSK3 $\beta$ pathway. Phosphorylated and thereby activated Akt was found to be highly elevated in FAT10 overexpressing cells, 
accompanied by an increase in the amount of phosphorylated and thus inactivated GSK3 $\beta$ and a stabilization of $\beta$-catenin (Liu et al., 2014). An increase in $\beta$-catenin in HCC was already reported earlier and it had been shown that this contributes to degradation of E-cadherin and induction of EMT (Nejak-Bowen and Monga, 2011). In summary, FAT10 seems to elicit its stabilizing effects onto $\beta$-catenin leading to cell migration, invasion and metastasis of HCC in two ways (Fig. 4). In a first line, FAT10 directly interacts with $\beta$-catenin which prevents its ubiquitylation and proteasomal degradation leading to increased $\beta$-catenin levels and thus to increased expression of downstream targets such as HOXB9. In a second line, overexpression of FAT10 elevates the amount of active Akt, which in turn phosphorylates GSK3 $\beta$, rendering it inactive which in turn stabilizes $\beta$-catenin. The Akt-GSK3 $\beta$ signaling pathway is therefore another intriguing mechanism of how FAT10 exerts its carcinogenic effects in liver carcinoma.

\section{4. $\operatorname{Smad} 2$}

Still another downstream target of FAT10 was identified by investigating the contribution of FAT10 upregulation onto the development of glioma, a neuroepithelial tumor arising from glia cells and accounting for nearly half of all intracranial tumors (Filbin and Suva, 2016). Gliomas are classified into four grades (I-IV) with increasing severity. FAT10 is prominently expressed in gliomas, with the highest mRNA and protein expression in grade IV gliomas. The increase in FAT10 expression positively correlates with progression of the disease and FAT10-positive patients have the lowest survival rate (Dai et al., 2016; Yuan et al., 2012). Glioma cell lines stably overexpressing FAT10 displayed enhanced tumorigenesis in vitro because they developed significantly more spheres and possessed increasing migration and invasion capacity. When injected into nude mice, these cells developed larger tumors as compared to mock-transfected cells, implicating that FAT10 is also promoting glioma carcinogenesis. FAT10 seems to exert its effect on gliomagenesis via the transforming growth factor beta (TGF 1 1) signaling pathway. Whereas in normal cells TGF $\beta$ inhibits proliferation, it turns out to be an oncogenic factor in high grade glioma because it influences glioma proliferation, survival, migration, invasion and the escape from host immunity. Dimeric TGF $\beta$ binding to one of the TGF $\beta$ type I and II receptor dimers induces conformational changes and activates kinase domains of the receptors. This leads to the phosphorylation and activation of Smad2 and Smad3 transcription factors, which interact with Smad4 and accumulate in the nucleus where they regulate the transcription of TGF $\beta$ expression itself but also of genes e.g. involved in cell cycle regulation (Kaminska et al., 2013). Overexpression of FAT10 in the glioma cell line U251 led to significantly increased levels of phosphorylated Smad2 (p-Smad2) and of other cancer stem-cell markers such as CD133, OCT4, nestin, and Bmi-1. In turn, knockdown of FAT10 or the application of an inhibitor of the TGF $\beta$ signaling pathway (LY364947) reversed this effect. Although the exact mechanism of this function of FAT10 needs further clarification, it appears that aberrant FAT10 might influence the progression of glioma by enhancing the level of $\mathrm{p}$ Smad2 (Dai et al., 2016).

\section{Summary and future prospects}

Growing evidence speaks for a direct involvement of the ubiquitin-like modifier FAT10 in tumorigenesis. Recent data strongly suggests that FAT10 itself promotes carcinogenesis by directly increasing survival, proliferation, invasion, and metastasis formation of tumor cells as summarized in Fig. 1. Given that FAT10 is a potent signal for proteasomal degradation, it is remarkable that other than the FAT10ylation of p53 no covalent substrates of FAT10 conjugation with potential for tumor promotion (e.g. tumor suppressors or apoptosis inducers) have been described. Since FAT10 gets isopeptide linked to hundreds of proteins, this is clearly a field for future investigations. Another central open question is why FAT10, when ectopically overexpressed mainly in nontransformed cells, potently induces apoptosis while it seems to promote cell growth and survival in other cells, in particular in cancer derived cell lines. The strong increase in FAT10 expression in a number of different tumor types might be useful as a prognostic marker for the survival rate of the affected patients and for estimating the probability of recurrence. Potential therapeutic avenues aiming at FAT10-inducing pathways such as NF- $\mathrm{\kappa B} / \mathrm{STAT3}$ signaling as well as targeting the FAT10 conjugation pathway seem to hold promise in particular because the genetic ablation of FAT10 in mice does not cause severe phenotypes. A possible starting point for targeting the FAT10 conjugation pathway might be an inhibitor of the FAT10 E1 activating enzyme UBA6, developed by Gavin and colleagues, which was already successfully tested to inhibit ubiquitin activation by UBA6 (Gavin et al., 2012). However, since UBA6 is a bispecific E1 activating enzyme for FAT10 and ubiquitin, this option must be handled with care to minimize putative side effects. Targeting the ubiquitin-proteasome system in cancer is already clinically effective at least via inhibition of the proteasome while inhibitors or activators of E3 ligases or DUBs with a better perspective for selectivity are being developed (Weathington and Mallampalli, 2014). The search for FAT10-specific E3 ligases as well as for FAT10-specific deconjugating enzymes is still ongoing and the identification of these enzymes will enable investigations on whether targeting of these enzymes will provide new options for the development of future pharmaceuticals against cancer.

\section{Conflict of interest}

The authors declare no conflict of interest.

\section{Acknowledgements}

This work was funded by grants from the Collaborative Research Center 969, Project C01, of the German Research Foundation and by grant Nrs. 855 and 1029 from the Velux foundation. The funding sources had no influence on writing of this article and on the publication decision.

\section{References}

Abate-Shen, C., 2002. Deregulated homeobox gene expression in cancer: cause or consequence? Nat. Rev. Cancer 2 (10), 777-785.

Aichem, A., Pelzer, C., Lukasiak, S., Kalveram, B., Sheppard, P.W., Rani, N., Schmidtke, G., Groettrup, M., 2010. USE1 is a bispecific conjugating enzyme for ubiquitin and FAT10, which FAT10ylates itself in cis. Nat. Commun. 1, 13.

Aichem, A., Kalveram, B., Spinnenhirn, V., Kluge, K., Catone, N., Johansen, T., Groettrup, M., 2012. The proteomic analysis of endogenous FAT10 substrates identifies p62/SQSTM1 as a substrate of FAT10ylation. J. Cell Sci. 125, 4576-4585.

Aichem, A., Catone, N., Groettrup, M., 2014. Investigations into the auto-FAT10ylation of the bispecific E2 conjugating enzyme UBA6-specific E2 enzyme 1. FEBS J. 281 (7), 1848-1859.

Amit, S., Hatzubai, A., Birman, Y., Andersen, J.S., Ben-Shushan, E., Mann, M., Ben-Neriah, Y., Alkalay, I., 2002. Axin-mediated CKI phosphorylation of beta-catenin at Ser 45: a molecular switch for the Wnt pathway. Genes Dev. 16 (9), 1066-1076.

Balkwill, F., 2009. Tumour necrosis factor and cancer. Nat. Rev. Cancer 9 (5), 361-371.

Bates, E.E., Ravel, O., Dieu, M.C., Ho, S., Guret, C., Bridon, J.M., Ait-Yahia, S., Briere, F., Caux, C., Banchereau, J., Lebecque, S., 1997. Identification and analysis of a novel member of the ubiquitin family expressed in dendritic cells and mature B cells. Eur. J. Immunol. 27 (10), 2471-2477.

Bett, J.S., Kanuga, N., Richet, E., Schmidtke, G., Groettrup, M., Cheetham, M.E., van der Spuy, J., 2012. The inherited blindness protein AIPL1 regulates the ubiquitin-like FAT10 pathway. PLoS One 7 (2), e30866. 
Bialas, J., Groettrup, M., Aichem, A., 2015. Conjugation of the ubiquitin activating enzyme UBE1 with the ubiquitin-like modifier FAT10 targets it fo proteasomal degradation. PLoS One 3, e0120329.

Bosch-Barrera, J., Menendez, J.A., 2015. Silibinin and STAT3: a natural way of targeting transcription factors for cancer therapy. Cancer Treat. Rev. 41 (6) 540-546.

Buerger, S., Herrmann, V.L., Mundt, S., Trautwein, N., Groettrup, M., Basler, M., 2015. The ubiquitin-like modifier FAT10 is selectively expressed in medullary thymic epithelial cells and modifies T cell selection. J. Immunol. 195 (9), $4106-4116$.

Canaan, A., Yu, X., Booth, C.J., Lia, J., Lazar, I., Gamfi, S.L., Castille, K., Kohya, N., Nakayama, Y., Liu, Y.C., Eynon, E., Flavell, R., Weissman, S.M., 2006. FAT10/diubiquitin-like protein-deficient mice exhibit minimal phenotypic differences. Mol. Cell Biol. 26 (13), 5180-5189.

Canaan, A., Defuria, J., Perelman, E., Schultz, V., Seay, M., Tuck, D., Flavell, R.A., Snyder, M.P., Obin, M.S., Weissman, S.M., 2014. Extended lifespan and reduced adiposity in mice lacking the FAT10 gene. Proc. Natl. Acad. Sci. U. S. A. 111 (14) 5313-5318.

Chen, D., Dou, Q.P., 2010. The ubiquitin-proteasome system as a prospective molecular target for cancer treatment and prevention. Curr. Protein Pept. Sci. 11 (6), 459-470

Chen, J., Yang, L., Chen, H., Yuan, T., Liu, M., Chen, P., 2014. Recombinant adenovirus encoding FAT10 small interfering RNA inhibits HCC growth in vitro and in vivo. Exp. Mol. Pathol. 96 (2), 207-211.

Chiu, Y.H., Sun, Q., Chen, Z.J., 2007. E1-L2 activates both ubiquitin and FAT10. Mol. Cell 27 (6), 1014-1023.

Choi, Y., Kim, J.K., Yoo, J.Y., 2014. NFkappaB and STAT3 synergistically activate the expression of FAT10, a gene counteracting the tumor suppressor p53. Mol. Oncol. 8 (3), 642-655.

Ciechanover, A., Heller, H., Katz-Etzion, R., Hershko, A., 1981. Activation of the heat-stable polypeptide of the ATP-dependent proteolytic system. Proc. Natl. Acad. Sci. U. S. A. 78 (2), 761-765.

Ciechanover, A., 1998. The ubiquitin-proteasome pathway: on protein death and cell life. EMBO J. 17 (24), 7151-7160.

Dai, B., Zhang, Y., Zhang, P., Pan, C., Xu, C., Wan, W., Wu, Z., Zhang, J., Zhang, L., 2016. Upregulation of p-Smad2 contributes to FAT10-induced oncogenic activities in glioma. Tumour Biol., http://dx.doi.org/10.1007/s13277-015-4739-6 (in press).

de La Coste, A., Romagnolo, B., Billuart, P., Renard, C.A., Buendia, M.A., Soubrane, O. Fabre, M., Chelly, J., Beldjord, C., Kahn, A., Perret, C., 1998. Somatic mutations of the beta-catenin gene are frequent in mouse and human hepatocellular carcinomas. Proc. Natl. Acad. Sci. U. S. A. 95 (15), 8847-8851.

Ebstein, F., Lange, N., Urban, S., Seifert, U., Kruger, E., Kloetzel, P.M., 2009. Maturation of human dendritic cells is accompanied by functional remodelling of the ubiquitin-proteasome system. Int. J. Biochem. Cell Biol. 41 (5), 1205-1215.

Ebstein, F., Lehmann, A., Kloetzel, P.M., 2012. The FAT10- and ubiquitin-dependent degradation machineries exhibit common and distinct requirements for MHC class I antigen presentation. Cell Mol. Life Sci. CMLS 69 (14), 2443-2454.

Fan, W., Cai, W., Parimoo, S., Schwarz, D.C., Lennon, G.G., Weissman, S.M., 1996. Identification of seven new human MHC class I region genes around the HLA-F locus. Immunogenetics 44 (2), 97-103.

Filbin, M.G., Suva, M.L., 2016. Glioma genomics and epigenomics: arriving at the start and knowing it for the first time. Annu. Rev. Pathol. 11, 497-521.

Finley, D., 2009. Recognition and processing of ubiquitin-protein conjugates by the proteasome. Annu. Rev. Biochem. 78, 477-513.

French, S.W., Bardag-Gorce, F., Li, J., French, B.A., Oliva, J., 2010a. Mallory-Denk body pathogenesis revisited. World J. Hepatol. 2 (8), 295-301.

French, S.W., Oliva, J., French, B.A., Li, J., Bardag-Gorce, F., 2010b. Alcohol, nutrition and liver cancer: role of Toll-like receptor signaling. World J. Gastroenterol. 16 (11), 1344-1348.

Gao, Z., Wang, X., Wu, K., Zhao, Y., Hu, G., 2010. Pancreatic stellate cells increase the invasion of human pancreatic cancer cells through the stromal cell-derived factor-1/CXCR4 axis. Pancreatology 10 (2-3), 186-193.

Gao, Y., Theng, S.S., Zhuo, J., Teo, W.B., Ren, J., Lee, C.G., 2014. FAT10, an biquitin-like protein, confers malignant properties in non-tumorigenic and tumorigenic cells. Carcinogenesis 35 (4), 923-934.

Gao, Y., Theng, S.S., Mah, W.C., Lee, C.G., 2015. Silibinin down-regulates FAT10 and modulate TNF-alpha/IFN-gamma-induced chromosomal instability and apoptosis sensitivity. Biol. Open 4 (8), 961-969.

Gavin, J.M., Chen, J.J., Liao, H., Rollins, N., Yang, X., Xu, Q., Ma, J., Loke, H.K., Lingaraj, T., Brownell, J.E., Mallender, W.D., Gould, A.E., Amidon, B.S., Dick, L.R. 2012. Mechanistic studies on activation of ubiquitin and di-ubiquitin-like protein, FAT10, byubiquitin-like modifier activating enzyme 6, Uba6. J. Biol Chem. 287 (19), 15512-15522.

Gong, P., Canaan, A., Wang, B., Leventhal, J., Snyder, A., Nair, V., Cohen, C.D., Kretzler, M., D'Agati, V., Weissman, S., Ross, M.J., 2010. The ubiquitin-like protein FAT10 mediates NF-kappaB activation. J. Am. Soc. Nephrol. 21 (2), 316-326.

Grivennikov, S.I., Greten, F.R., Karin, M., 2010. Immunity, inflammation, and cancer Cell 140 (6), 883-899.

Groettrup, M., Pelzer, C., Schmidtke, G., Hofmann, K., 2008. Activating the ubiquitin family: UBA6 challenges the field. Trends Biochem. Sci. 33 (5), 230-237.

Gu, X., Zhao, F., Zheng, M., Fei, X., Chen, X., Huang, S., Xie, Y., Mao, Y., 2007. Cloning and characterization of a gene encoding the human putative ubiquitin conjugating enzyme E2Z (UBE2Z). Mol. Biol. Rep. 34 (3), 183-188.

Gudkov, A.V., Gurova, K.V., Komarova, E.A., 2011. Inflammation and p53: a tale of two stresses. Genes Cancer 2 (4), 503-516.
Haglund, K., Dikic, I., 2005. Ubiquitylation and cell signaling. EMBO J. 24 (19), 3353-3359

Han, T., Liu, Z., Li, H., Xie, W., Zhang, R., Zhu, L., Guo, F., Han, Y., Sheng, Y., Xie, X., 2015. High expression of UBD correlates with epirubicin resistance and indicates poor prognosis in triple-negative breast cancer. Onco Targets Ther. 8 , 1643-1649.

Hatzis, P., van der Flier, L.G., van Driel, M.A., Guryev, V., Nielsen, F., Denissov, S., Nijman, J., Koster, J., Santo, E.E., Welboren, W., Versteeg, R., Cuppen, E., van de Wetering, M., Clevers, H., Stunnenberg, H.G., 2008. Genome-wide pattern of TCF7L2/TCF4 chromatin occupancy in colorectal cancer cells. Mol. Cell. Biol. 28 (8), 2732-2744

Heinrich, E.L., Lee, W., Lu, J., Lowy, A.M., Kim, J., 2012. Chemokine CXCL12 activates dual CXCR4 and CXCR7-mediated signaling pathways in pancreatic cancer cells. J. Transl. Med. 10, 68 .

Hipp, M.S., Kalveram, B., Raasi, S., Groettrup, M., Schmidtke, G., 2005. FAT10, a ubiquitin- independent signal for proteasomal degradation. Mol. Cell. Biol. 25 (9), 3483-3491.

Hochstrasser, M., 2009. Origin and function of ubiquitin-like proteins. Nature 458 (7237), 422-429.

Hoesel, B., Schmid, J.A., 2013. The complexity of NF-kappaB signaling in inflammation and cancer. Mol. Cancer 12, 86 .

Hong, Y.K., Foreman, K., Shin, J.W., Hirakawa, S., Curry, C.L., Sage, D.R., Libermann, T., Dezube, B.J., Fingeroth, J.D., Detmar, M., 2004. Lymphatic reprogramming o blood vascular endothelium by Kaposi sarcoma-associated herpesvirus. Nat. Genet. 36 (7), 683-685.

Ji, F., Jin, X., Jiao, C.H., Xu, Q.W., Wang, Z.W., Chen, Y.L., 2009. FAT10 level in human gastric cancer and its relation with mutant p53 level, lymph node metastasis and TNM staging. World J. Gastroenterol. 15 (18), 2228-2233.

Jin, J., Li, X., Gygi, S.P., Harper, J.W., 2007. Dual E1 activation systems for ubiquitin differentially regulate E2 enzyme charging. Nature 447 (7148), 1135-1138.

Kalveram, B., Schmidtke, G., Groettrup, M., 2008. The ubiquitin-like modifier FAT10 interacts with HDAC6 and localizes to aggresomes under proteasome inhibition. J. Cell Sci. 121 (Pt 24), 4079-4088.

Kaminska, B., Kocyk, M., Kijewska, M., 2013. TGF beta signaling and its role in glioma pathogenesis. Adv. Exp. Med. Biol. 986, 171-187.

Karin, M., 2006. Nuclear factor-kappaB in cancer development and progression. Nature 441 (7092), 431-436.

Katoh, M., Katoh, M., 2010. Integrative genomic analyses of CXCR4: transcriptional regulation of CXCR4 based on TGFbeta, Nodal, Activin signaling and POU5F1, FOXA2, FOXC2, FOXH1, SOX17, and GFI1 transcription factors. Int. J. Oncol. 36 (2), 415-420,

Kerscher, O., Felberbaum, R., Hochstrasser, M., 2006. Modification of proteins by ubiquitin and ubiquitin-like proteins. Annu. Rev, Cell Dev, Biol. 22, 159-180.

Kito, K., Yeh, E.T., Kamitani, T., 2001. NUB1, a NEDD8-interacting protein, is induced by interferon and down-regulates the NEDD8 expression. J. Biol. Chem. 276 (23), 20603-20609.

Lai, T.Y., Su, C.C., Kuo, W.W., Yeh, Y.L., Kuo, W.H., Tsai, F.J., Tsai, C.H., Weng. Y.J. Huang, C.Y., Chen, L.M., 2011. Beta-catenin plays a key role in metastasis of human hepatocellular carcinoma. Oncol. Rep. 26 (2), 415-422.

Lara-Gonzalez, P., Westhorpe, F.G., Taylor, S.S., 2012. The spindle assembly checkpoint. Curr. Biol. CB 22, R966-R980.

Lee, C.G., Ren, J., Cheong, I.S., Ban, K.H., Ooi, L.L., Yong Ta, n.S., Kan, A., Nuchprayoon, I., Jin, R., Lee, K.H., Choti, M., Lee, L.A., 2003. Expression of the FAT10 gene is highly upregulated in hepatocellular carcinoma and other gastrointestinal and gynecological cancers. Oncogene 22 (17), 2592-2603.

Leng, L., Xu, C., Wei, C., Zhang, J., Liu, B., Ma, J., Li, N., Qin, W., Zhang, W., Zhang, C., Xing Zhai, L., Yang, F., Li, M., Jin, C., Yuan, Y., Xu, P., Qin, J., Xie, H., He, F., Wang, J., 2014. A proteomics strategy for the identification of FAT10-modified sites by mass spectrometry. J. Proteome Res. 13 (1), 268-276.

Levine, A.J., 1997. p53, the cellular gatekeeper for growth and division. Cell 88 (3), 323-331.

Li, T., Santockyte, R., Yu, S., Shen, R.F., Tekle, E., Lee, C.G., Yang, D.C., Chock, P.B., 2011. FAT10 modifies p53 and upregulates its transcriptional activity. Arch. Biochem. Biophys. 509 (2), 164-169.

Liang, Z., Yoon, Y., Votaw, J., Goodman, M.M., Williams, L., Shim, H., 2005. Silencing of CXCR4 blocks breast cancer metastasis. Cancer Res. 65 (3), 967-971.

Lim, C.B., Zhang, D., Lee, C.G., 2006. FAT10, a gene up-regulated in various cancers, is cell-cycle regulated. Cell Div. 1, 20.

Liu, Y.C., Pan, J., Zhang, C., Fan, W., Collinge, M., Bender, J.R., Weissman, S.M., 1999. A MHC-encoded ubiquitin-like protein (FAT10) binds noncovalently to the spindle assembly checkpoint protein MAD2. Proc. Natl. Acad. Sci. U. S. A. 96 (8), 4313-4318.

Liu, L., Dong, Z., Liang, J., Cao, C., Sun, J., Ding, Y., Wu, D., 2014. As an independent prognostic factor, FAT10 promotes hepatitis B virus-related hepatocellular carcinoma progression via Akt/GSK3beta pathway. Oncogene 33 (7), 909-920.

Lukasiak, S., Schiller, C., Oehlschlaeger, P., Schmidtke, G., Krause, P., Legler, D.F. Autschbach, F., Schirmacher, P., Breuhahn, K., Groettrup, M., 2008. Proinflammatory cytokines cause FAT10 upregulation in cancers of liver and colon. Oncogene 27 (46), 6068-6074.

Manning, B.D., Cantley, L.C., 2007. AKT/PKB signaling: navigating downstream. Cell 129 (7), 1261-1274

Merbl, Y., Refour, P., Patel, H., Springer, M., Kirschner, M.W., 2013. Profiling of ubiquitin-like modifications reveals features of mitotic control. Cell 152 (5), $1160-1172$

Musacchio, A., Salmon, E.D., 2007. The spindle-assembly checkpoint in space and time. Nat. Rev. Mol. Cell Biol. 8 (5), 379-393. 
Nejak-Bowen, K.N., Monga, S.P., 2011. Beta-catenin signaling, liver regeneration and hepatocellular cancer: sorting the good from the bad. Semin. Cancer Biol. 21 (1), 44-58.

Nguyen, N.T., Now, H., Kim, W.J., Kim, N., Yoo, J.Y., 2016. Ubiquitin-like modifier FAT10 attenuates RIG-I mediated antiviral signaling by segregating activated RIG-I from its signaling platform. Sci. Rep. 6, 23377.

Oliva, J., Bardag-Gorce, F., French, B.A., Li, J., McPhaul, L., Amidi, F., Dedes, J., Habibi, A., Nguyen, S., French, S.W., 2008. Fat10 is an epigenetic marker for liver preneoplasia in a drug-primed mouse model of tumorigenesis. Exp. Mol. Pathol. 84 (2), 102-112.

Pelzer, C., Kassner, I., Matentzoglu, K., Singh, R.K., Wollscheid, H.P., Scheffner, M., Schmidtke, G., Groettrup, M., 2007. UBE1L2, a novel E1 enzyme specific for ubiquitin. J. Biol. Chem. 282 (32), 23010-23014.

Peng, X., Shao, J., Shen, Y., Zhou, Y., Cao, Q., Hu, J., He, W., Yu, X., Liu, X., Marian, A.J., Hong, K., 2013. FAT10 protects cardiac myocytes against apoptosis. J. Mol. Cell Cardiol. 59, 1-10

Polakis, P., 2000. Wnt signaling and cancer. Genes Dev. 14 (15), 1837-1851.

Polakis, P., 2012. Wnt signaling in cancer. Cold Spring Harb. Perspect. Biol. 4 (5).

Qing, X., French, B.A., Oliva, J., French, S.W., 2011. Increased expression of FAT10 in colon benign, premalignant and malignant epithelial neoplasms. Exp. Mol. Pathol. 90 (1), 51-54

Raasi, S., Schmidtke, G., de Giuli, R., Groettrup, M., 1999. A ubiquitin-like protein which is synergistically inducible by interferon-gamma and tumor necrosis factor-alpha. Eur. J. Immunol. 29 (12), 4030-4036.

Raasi, S., Schmidtke, G., Groettrup, M., 2001. The ubiquitin-like protein FAT10 forms covalent conjugates and induces apoptosis. J. Biol. Chem. 276 (38), 35334-35343.

Rani, N., Aichem, A., Schmidtke, G., Kreft, S.G., Groettrup, M., 2012. FAT10 and NUB1L bind to the VWA domain of Rpn10 and Rpn1 to enable proteasome-mediated proteolysis. Nat. Commun. 3, 749 .

Ren, J., Kan, A., Leong, S.H., Ooi, L.L., Jeang, K.T., Chong, S.S., Kon, O.L., Lee, C.G. 2006. FAT10 plays a role in the regulation of chromosomal stability. J. Biol. Chem. 281 (16), 11413-11421.

Ren, J., Wang, Y., Gao, Y., Mehta, S.B., Lee, C.G., 2011. FAT10 mediates the effect of TNF-alpha in inducing chromosomal instability. J. Cell Sci. 124 (Pt 21), 3665-3675.

Reyes-Turcu, F.E., Ventii, K.H., Wilkinson, K.D., 2009. Regulation and cellular roles of ubiquitin-specific deubiquitinating enzymes. Annu. Rev. Biochem. 78 , 363-397.

Ross, M.J., Wosnitzer, M.S., Ross, M.D., Granelli, B., Gusella, G.L., Husain, M. Kaufman, L., Vasievich, M., D’Agati, V.D., Wilson, P.D., Klotman, M.E., Klotman, P.E., 2006. Role of ubiquitin-like protein FAT10 in epithelial apoptosis in renal disease. J. Am. Soc Nephrol 17 (4), 996-1004.

Schliehe, C., Bitzer, A., van den Broek, M., Groettrup, M., 2012. Stable antigen is most effective for eliciting CD8+ T-cell responses after DNA vaccination and infection with recombinant vaccinia virus in vivo. J. Virol. 86 (18), 9782-9793.

Schmidtke, G., Kalveram, B., Weber, E., Bochtler, P., Lukasiak, S., Hipp, M.S. Groettrup, M., 2006. The UBA domains of NUB1L are required for binding but not for accelerated degradation of the ubiquitin-like modifier FAT10. J. Biol. Chem. 281 (29), 20045-20054.

Schmidtke, G., Kalveram, B., Groettrup, M., 2009. Degradation of FAT10 by the 26S proteasome is independent of ubiquitylation but relies on NUB1L. FEBS Lett. $583(3), 591-594$

Schmidtke, G., Aichem, A., Groettrup, M., 2014. FAT10ylation as a signal for proteasomal degradation. Biochim. Biophys. Acta 1843 (1), 97-102.

Singh, R.P., Mallikarjuna, G.U., Sharma, G., Dhanalakshmi, S., Tyagi, A.K., Chan, D.C., Agarwal, C., Agarwal, R., 2004. Oral silibinin inhibits lung tumor growth in athymic nude mice and forms a novel chemocombination with doxorubicin targeting nuclear factor kappaB-mediated inducible chemoresistance. Clin. Cancer Res. 10 (24), 8641-8647.
Snyder, A Alsauskas, Z Gong P. Rosenstiel, P.E., Klotman, M.E., Klotman, P.E. Ross, M.J., 2009. FAT10: a novel mediator of Vpr-induced apoptosis in human immunodeficiency virus-associated nephropathy. J. Virol. 83 (22), 11983-11988.

Spinnenhirn, V., Farhan, H., Basler, M., Aichem, A., Canaan, A., Groettrup, M., 2014 The ubiquitin-like modifier FAT10 decorates autophagy-targeted Salmonella and contributes to Salmonella resistance in mice. J. Cell Sci. 127 (22), 4883-4893.

Sun, G.H., Liu, Y.D., Yu, G., Li, N., Sun, X., Yang, J., 2014. Increased FAT10 expression is related to poor prognosis in pancreatic ductal adenocarcinoma. Tumour Biol. 35 (6), 5167-5171.

Tarnowski, M., Grymula, K., Reca, R., Jankowski, K., Maksym, R., Tarnowska, J., Przybylski, G., Barr, F.G., Kucia, M., Ratajczak, M.Z., 2010. Regulation of expression of stromal-derived factor-1 receptors: CXCR4 and CXCR7 in human rhabdomyosarcomas. Mol. Cancer Res. MCR 8 (1), 1-14.

Theng, S.S., Wang, W., Mah, W.C., Chan, C., Zhuo, J., Gao, Y., Qin, H., Lim, L., Chong, S.S., Song, J., Lee, C.G., 2014. Disruption of FAT10-MAD2 binding inhibits tumor progression. Proc. Natl. Acad. Sci. U. S. A. 111 (49), E5282-5291.

Tu, Y, Chen, C. Pan, J. Xu, J., Zhou, Z.G, Wang, C.Y, 2012. The Ubiquitin Proteasome Pathway (UPP) in the regulation of cell cycle control and DNA damage repair and its implication in tumorigenesis. Int. J. Clin. Exp. Pathol. 5 (8), 726-738.

Tyagi, A., Agarwal, C., Dwyer-Nield, L.D., Singh, R.P., Malkinson, A.M., Agarwal, R., 2012. Silibinin modulates TNF-alpha and IFN-gamma mediated signaling to regulate COX2 and iNOS expression in tumorigenic mouse lung epithelial LM2 cells. Mol. Carcinog. 51 (10), 832-842.

Wake, M.S., Watson, C.J., 2015. STAT3 the oncogene-still eluding therapy? FEBS J 282 (14), 2600-2611.

Wang, H., Wang, H.S., Zhou, B.H., Li, C.L., Zhang, F., Wang, X.F., Zhang, G., Bu, X.Z., Cai, S.H., Du, J., 2013. Epithelial-mesenchymal transition (EMT) induced by TNF-alpha requires AKT/GSK-3beta-mediated stabilization of snail in colorectal cancer. PLoS One 8 (2), e56664.

Weathington, N.M., Mallampalli, R.K., 2014. Emerging therapies targeting the ubiquitin proteasome system in cancer. J. Clin. Invest. 124 (1), 6-12.

Wolberger, C., 2014. Mechanisms for regulating deubiquitinating enzymes. Prot. Sci. 23 (4), 344-353.

Xu, P., Duong, D.M., Seyfried, N.T., Cheng, D., Xie, Y., Robert, J., Rush, J., Hochstrasser, M., Finley, D., Peng, J., 2009. Quantitative proteomics reveals the function of unconventional ubiquitin chains in proteasomal degradation. Cell 137 (1), 133-145

Yuan, J., Tu, Y., Mao, X., He, S., Wang, L., Fu, G., Zong, J., Zhang, Y., 2012. Increased expression of FAT10 is correlated with progression and prognosis of human glioma. Pathol. Oncol. Res. POR 18 (4), 833-839.

Yuan, R., Wang, K., Hu, J., Yan, C., Li, M., Yu, X., Liu, X., Lei, J., Guo, W., Wu, L., Hong K., Shao, J., 2014. Ubiquitin-like protein FAT10 promotes the invasion and metastasis of hepatocellular carcinoma by modifying beta-catenin degradation. Cancer Res. 74 (18), 5287-5300.

Zamarron, B.F., Chen, W., 2011. Dual roles of immune cells and their factors in cancer development and progression. Int. J. Biol. Sci. 7 (5), 651-658.

Zhang, D.W., Jeang, K.T., Lee, C.G., 2006. p53 negatively regulates the expression of FAT10, a gene upregulated in various cancers. Oncogene 25 (16), 2318-2327.

Zhao, S., Jiang, T., Tang, H., Cui, F., Liu, C., Guo, F., Lu, H., Xue, Y., Jiang, W., Peng, Z. Yan, D., 2015. Ubiquitin D is an independent prognostic marker for survival in stage IIB-IIC colon cancer patients treated with 5-fluoruracil-based adjuvant chemotherapy. J. Gastroenterol. Hepatol. 30 (4), 680-688.

Zheng, K., Li, H.Y., Su, X.L., Wang, X.Y., Tian, T., Li, F., Ren, G.S., 2010. Chemokine receptor CXCR7 regulates the invasion, angiogenesis and tumor growth of human hepatocellular carcinoma cells. J. Exp. Clin. Cancer Res. CR 29, 31. 